\title{
AWARENESS OF CHEMOTHERAPY SIDE EFFECTS AND ATTITUDE TOWARDS CHEMOTHERAPY USE AMONG CANCER PATIENTS ATTENDING ONCOLOGY CLINIC AT BUGANDO MEDICAL CENTRE, IN MWANZA, NORTHERN TANZANIA
}

\author{
*Deogratias M Katabalo ${ }^{1,3}$, Raphael Matinde ${ }^{1}$, Stanley Mwita ${ }^{1}$, Karoli Marwa ${ }^{2}$, Nestory Masalu ${ }^{3}$ \\ ${ }^{1}$ School of Pharmacy, Catholic University of Health and Allied Sciences (CUHAS), P.O. Box 1464, Mwanza, Tanzania \\ ${ }^{2}$ Department of Pharmacology, Catholic University of Health and Allied Sciences (CUHAS), P.O. Box 1464, Mwanza, Tanzania \\ ${ }^{3}$ Department of Oncology, Bugando Medical centre (BMC), P.O. Box 1370, Mwanza, Tanzania
}

\begin{abstract}
Background: Chemotherapy is the use of drugs to kill cancer cells. Because cancer cells generally grow and divide faster than normal cells, they are more susceptible to the action of these drugs. However, damage to healthy cells is unavoidable, and this damage accounts for the side effects linked to these drugs.

Methodology: A cross section study was conducted at oncology department in Bugando Medical Centre. A sample of 216 people was recruited into the study. The data was collected by using the pre-constructed questionnaire. After data collection, the data was transferred into SPSS version 20 and analyzed.

Results: The study found that $88(40.7 \%)$ of the respondents were aware of the chemotherapy side effects whereby majority, 68 $(77.3 \%)$ of these had been informed by the doctor. Most of the respondents, $197(92.1 \%)$ had a positive attitude towards the use of chemotherapy.

Conclusion: There is still poor awareness of the chemotherapy side effects among the cancer patients attending and receiving chemotherapy in oncology department at Bugando Medical Centre. However, most of the respondents had positive attitude towards the use of chemotherapy
\end{abstract}

Keywords: Awareness, attitude, chemotherapy, side effects and Tanzania.

Article Info: Received 15 Aug, 2018; Review Completed 07 Sep 2018; Accepted 08 Sep 2018; Available online 15 Sep 2018

Cite this article as:

Katabalo DM, Matinde R, Mwita S, Marwa K, Masalu N, Awareness of Chemotherapy side effects and Attitude towards Chemotherapy use among Cancer Patients attending Oncology Clinic at Bugando Medical Centre, in Mwanza, Northern Tanzania, Journal of Drug Delivery and Therapeutics. 2018; 8(5):448-454 DOI: $\underline{\text { http://dx.doi.org/10.22270/jddt.v8i5.1900 }}$

*Address for Correspondence:

Deogratias M Katabalo, School of Pharmacy, Catholic University of Health and Allied Sciences (CUHAS), P.O.Box 1464, MwanzaTanzania

\section{INTRODUCTION}

Cancer, also known as a malignant tumor or malignant neoplasm is a group of diseases involving abnormal cell growth with the potential to invade or spread to other parts of the body ${ }^{1}$.

Cancers as a group of diseases account for approximately $13 \%$ of all deaths each year worldwide, with the most common being: lung cancer (1.4 million deaths), stomach cancer (740,000 deaths), liver cancer (700,000 deaths), colorectal cancer (610,000 deaths), and breast cancer $(460,000 \text { deaths })^{2}$. This makes invasive cancer the leading cause of death in the developed world and the second leading cause of death in the developing world. ${ }^{3}$

Africa had 715,000 new cases of cancer in 2008 and 542,000 deaths from cancer. This number is projected to $1.28 \mathrm{M}$ new cases and 970,000 deaths by 2030 which is nearly double of the current situation ${ }^{4}$.

In Tanzania, there were only two cancer treatment centers in 2002 to serve the entire population of 46 
million people. These two clinics have capacity to serve only about 5,000 of the estimated 30,000 new cancer cases each year. For many of the patients who manage to receive treatment, their cancers have already metastasized to the point that administering palliative care becomes the only remaining option ${ }^{5}$.

Use of chemotherapy drugs is the cornerstone modality of the management of various types of cancers. These powerful medications circulate in the bloodstream and directly damage the cells that are actively growing. Because cancer cells generally grow and divide faster than normal cells, they are more susceptible to the action of these drugs. However, damage to healthy cells is unavoidable, and this damage accounts for the side effects linked to these drugs ${ }^{6}$.

Chemotherapy drugs are also used as an adjuvant treatment (treatment given after surgery or radiation therapy) to kill any cancer cells that remains. It may also be used as neo-adjuvant therapy (treatment given before surgery or radiation therapy to shrink tumors). For cancers of the blood or lymph system, such as leukemia and lymphoma, chemotherapy may be the only treatment given. In addition, chemotherapy is used to treat recurrent cancer (cancer that comes back after treatment) or metastatic cancer (cancer that has spread to other parts of the body) ${ }^{7}$

The goals of treatment with chemotherapy drugs depend on the type of cancer and how much it has spread. In some situations, the primary goal of chemotherapy is to eliminate cancer cells and prevent recurrence. If it is not possible to eliminate the cancer cells, chemotherapy may be used to control the cancer by slowing its growth and/or to reduce symptoms caused by the cancer, ${ }^{\mathbf{6}, \mathbf{8}}$.

The side effects associated with chemotherapy are so noxious that result into poor adherence, poor quality of life, morbidity or even mortality ${ }^{9}$. Early education and information to the patients on these side effects helps to manage and cope with them and improve the quality of life ${ }^{10}$.

\section{METHODS}

\section{Study Area}

This study was conducted at the oncology clinic in the oncology department at Bugando Medical Center. This is the largest referral hospital in the Lake zone of north western Tanzania that serves all cancer cases including those referred from other hospitals. Furthermore, this hospital is a care to 8 regions harboring around 14 million citizens of Tanzania. All outpatients and inpatient cancer patients are seen at the oncology clinic; therefore this was the most feasible study site to access the targeted study population.

\section{Study Design}

A cross sectional design was employed. This involved primary data collection through interviewing the consented participants.

\section{Study population}

The study involved adult individuals aged 18 years or above who attend and receive chemotherapy treatment in oncology unit at Bugando Medical Centre.

\section{The sample size determination}

The estimated prevalence rate of cancer patients receiving chemotherapy at Bugando Medical Centre is $83 \%$ according to the hospital oncology department cancer registry ${ }^{11}$. Therefore, the prevalence of $83 \%$ was used in the estimation of the sample size using Fischer's formula:

$$
\mathrm{n}=\mathrm{Z}^{2} \times \mathrm{P}(1-\mathrm{P}) / \mathrm{d}^{2}
$$

Where $n=$ sample size

$\mathrm{P}=$ estimated prevalence rate of adherence to chemotherapy treatment $=83 \%$

$\mathrm{Z}=1.96$ which is $\mathrm{Z}$-value corresponding to a significance level of 0.05

$\mathrm{d}=0.05$ which is the desired degree of accuracy for the study.

Hence, $n=1.96^{2} \times 0.83 \times(1-0.83) / 0.05^{2}=216$

Sample size $=216$ people.

\section{Sampling method and recruitment of participants}

The participant was first recruited using eligibility assessment form through systematic random sampling method whereby every $2^{\text {nd }}$ patient leaving the consultation room at the oncology clinic, who met the inclusion criteria and signed an informed consent, was enrolled into the study.

\section{Data collection tools}

Pre-constructed semi-structured questionnaire was used to collect information from participants on the issues of knowledge and altitude towards chemotherapy side effects.

\section{Ethical Consideration}

The study started upon approval from the CUHAS/BMC Ethics and Research Committee and permission from BMC Oncology department. Written informed consent was sought from all participants first before administering the study tools. All participants were required to fully understand the study, know their rights and voluntarily sign the informed consent before participation. Participants were free to ask any questions regarding the study, their rights and seek any clarification. All information was kept confidential.

\section{Data Management}

Raw data was collected using study tools and entered into a password protected Microsoft excel (version 2007) Database and then exported to SPSS version 20 for analysis. Before and after analysis, the filled questionnaires were stored in a lockable cabinet by a researcher. They then got moved to a lockable cabinet in the statistician's office during data entry and analysis. 
Upon completion of Data entry, hard copy forms were compared with the entered data to identify errors and corrections made appropriately.

\section{RESULTS}

The study recruited 216 people, the number which was equivalent to the pre-estimated sample size. These were the individuals at the age of 18 years or above who had started the chemotherapy and consented to be part of the study.

\section{Social demographic characteristics of participants}

The study included the people of different social demographic characteristics as shown in the table 1 below.

Table 1: Social- demographic characteristics

\begin{tabular}{|l|l|l|}
\hline Variable & Frequency & Percentages (\%) \\
\hline Age & & \\
$18-30$ & 28 & 13 \\
$31-40$ & 38 & 17.6 \\
$41-50$ & 60 & 27.8 \\
$51-60$ & 56 & 25.9 \\
60+ & 34 & 15.7 \\
Sex & 102 & \\
Male & 114 & 47.2 \\
Female & & 52.8 \\
Marital status & 31 & \\
$\quad$ Single & 143 & 14.4 \\
Married & 9 & 66.2 \\
Divorced & 33 & 41.7 \\
Widowed & 15.3 \\
Occupation & 113 & \\
$\quad$ Peasants & 13 & 52.3 \\
Business & 24 & 6 \\
Employed & 13 & 11.1 \\
Self-employment & 53 & 6 \\
Unemployed & & 24.5 \\
Educational level. & 151 & \\
$\quad$ Primary education & 46 & 69.9 \\
Secondary education & 6 & 21.3 \\
College/university & 13 & 27.7 \\
No education. & 136 & 6 \\
Religion & 68 & 63 \\
Christian & 12 & 31.5 \\
Muslim & 161 & 55.6 \\
Pagans & 55 & 74.5 \\
Residence & & 25.5 \\
Rural & & \\
Urban. & & \\
& & \\
\hline
\end{tabular}

\section{Finding of the study}

\section{Awareness of chemotherapy side effects}

To assess the awareness on chemotherapy side effects, the participants were interviewed if they had received any information regarding the side effects before the start of chemotherapy and it was found that 88 participants $(40.7 \%)$ were informed of chemotherapy and therefore aware of them while 128 people $(59.3 \%)$ denied to have been given any information before the commencement of chemotherapy.

Table 2: Pre-information on chemotherapy side effects

\begin{tabular}{|l|l|r|r|}
\hline Participant's Response & Frequency & \multicolumn{2}{|c|}{ Percent } \\
\hline & Aware & 88 & 40.7 \\
\cline { 2 - 4 } & Not aware & 128 & 59.3 \\
\cline { 2 - 4 } & Total & 216 & 100.0 \\
\hline
\end{tabular}

Furthermore, among those who said were informed of the side effects, it was found from that, majority of them were informed about occurrence of vomiting $85(96.6 \%)$ and alopecia $82(93.2 \%)$ table 3. 
Table 3: Side effects informed about

\begin{tabular}{|l|l|l|}
\hline Side effect & Frequency & Percentage \\
\hline Vomiting & 85 & 96.6 \\
Nausea & 62 & 70.5 \\
Alopecia & 82 & 93.2 \\
Diarrhoea & 25 & 28.4 \\
Fatigue & 62 & 70.5 \\
Pain & 28 & 31.8 \\
Bleeding & 4 & 4.5 \\
Anaemia & 12 & 13.6 \\
Immune-suppression & 2 & 2.3 \\
Loss of apatite & 7 & 8 \\
Infertility. & 0 & 0 \\
& & \\
\hline
\end{tabular}

Because cancer patients interact with various caregivers, the study went further to identify the informer of the side effects and it was found that, $68(77.3 \%)$ were informed by the doctor, $19(21.6 \%)$ were informed by the nurse and $1(1.2 \%)$ respondent said was informed by a friend. The study also revealed that, none of participants among the informed participants was informed by the pharmacist (Table 4)

Table 4: The informer of side effects

\begin{tabular}{|l|l|r|r|}
\hline \multicolumn{2}{|l|}{ Informer } & Frequency & \multicolumn{2}{|c|}{ Percent } \\
\hline \multirow{3}{*}{} & Doctor & 68 & 77.3 \\
\cline { 2 - 4 } & Nurse & 19 & 21.6 \\
\cline { 2 - 4 } & Friend & 1 & 1.1 \\
\hline
\end{tabular}

The respondents were also interviewed on whether or not they experienced any side effects after the start of chemotherapy and 191 respondents $(88.4 \%)$ said they experienced side effects following the commencement of chemotherapy whereby 25 respondents $(11.6 \%)$ said had not experienced any side effects. Among those who experienced side effects, the response was as shown in table 5 .

Table 5: Experience of side effects

\begin{tabular}{|l|l|l|}
\hline Side effects & frequency & Percent(\%). \\
\hline Vomiting & 160 & 83.8 \\
Nausea & 140 & 73.3 \\
Alopecia & 141 & 73.8 \\
Diarrhoea & 67 & 35.1 \\
Fatigue & 157 & 82.2 \\
Pain & 77 & 40.3 \\
Loss of apatite & 42 & 23 \\
Loss of libido & 4 & 2.1 \\
Bleeding. & 1 & 0.5 \\
Other side effects. & 5 & 2.6 \\
\hline
\end{tabular}

The action taken by those who experienced side effects also varied. Some went back to see the doctor, some tolerated while others stopped the medication at least at one occasion as depicted in the table 6 .

Table 6: Action taken when experienced side effects

\begin{tabular}{|l|l|l|}
\hline Action & Frequency & Percentage \\
\hline Went back to doctor & 66 & 34.6 \\
Tolerated & 70 & 36.6 \\
Stopped medications. & 55 & 28.8 \\
\hline
\end{tabular}

The participants were also interviewed if they had been informed and are aware of any medications which relieve the side effects. Only 8 respondents $(3.7 \%)$ acknowledged to be aware while the majority, 208
(96.3\%) denied to have been informed. The study went on to assess the association between awareness and different variables; the result was as shown in the table 7. 
Table 7: The association between awareness and other variables

\begin{tabular}{|l|l|l|}
\hline \multirow{2}{*}{ Variable } & \multicolumn{2}{|c|}{ Awareness. } \\
\cline { 2 - 3 } & Chi square value & P value. \\
\hline Age & 2.90 & 0.57 \\
Sex & 1.52 & 0.21 \\
Occupation & 9.18 & 0.06 \\
Level of education & 5.32 & 0.15 \\
Residence & 2.13 & 0.14 \\
Duration in chemotherapy & 17.72 & 0.01 \\
Action & 7.72 & 0.02 \\
Awareness of relief med. & 12.08 & 0.01 \\
attitude & 10.86 & 0.01 \\
\hline
\end{tabular}

There was a significant statistical association between participants being aware of the side effects and duration with which the patient has been on chemotherapy, action to take when side effects arise, awareness of side effects relieving medications and attitude towards the use of chemotherapy.

\section{Attitude towards chemotherapy use}

To determine the attitude towards the use of chemotherapy, the participants were asked to give the views towards the use. Most of respondents said chemotherapy was very helpful and they were ready to continue with chemotherapy. The results are shown in the table 8 .

Table 8: Attitude towards chemotherapy use

\begin{tabular}{|c|c|c|c|}
\hline \multicolumn{2}{|c|}{ Attitude. } & Frequency & Percent \\
\hline & Positive. & 197 & 91.2 \\
\cline { 2 - 4 } & Negative. & 19 & 8.8 \\
\hline Total & 216 & 100.0 \\
\hline
\end{tabular}

The associations between the attitude and different variables were determined and the results are shown in table 9 .

Table 9: The associations between attitude and other variables

\begin{tabular}{|l|l|l|}
\hline Variable. & Attitude. \\
\cline { 2 - 3 } & Chi square value & P value \\
\hline Age & 2.726 & 0.61 \\
Sex & 0.219 & 0.64 \\
Marital status & 1.382 & 0.71 \\
Occupation & 2.689 & 0.61 \\
Level of education & 1.768 & 0.62 \\
Religion & 7.714 & 0.42 \\
Residence & 4.840 & 0.52 \\
Duration in chemotherapy & 3.976 & 0.78 \\
Awareness of SE. & 10.864 & 0.01 \\
\hline
\end{tabular}

There was significant statistical association between participants being aware of the side effects and having positive attitude.

\section{DISCUSSION}

\section{Awareness on the chemotherapy side effects}

With regard to awareness on chemotherapy side effects, participants were interviewed to find out if they had received any information regarding the side effects. It was found that only 88 respondents $(40.7 \%)$ were aware of chemotherapy side effects before the start of chemotherapy. The lower rate of awareness among these participants would be attributed to lower number of health personnel working in this facility; therefore because of large burden of patients to health personnel, it would have made it difficult for patients to have enough time to receive all information. This rate was lower compared to study done by Julie Tompson, 2013 in Canada whereby the awareness was $79 \%{ }^{7,12}$. This was indeed lower than the required rate of $100 \%$ by the WHO which require all patients to be aware of the side effects before the start of chemotherapy ${ }^{\mathbf{1 3}}$. Other similar studies done on the awareness of side effects of chemotherapy drugs used in treatment of various types of cancers showed slightly higher rate of awareness $14,15,16$

Although $88(40.7 \%)$ of all participants were aware of chemotherapy side effects, majority of them were aware of vomiting $(85,96.6 \%)$, alopecia $(82,93.2 \%)$, nausea $(62,70.5 \%)$ and fatigue $(62,70.5 \%)$. This is probably because these are the side effects which occur immediately following the use of almost all chemotherapies and many patients are told about them by friends and primary care givers despite of expecting to be told by health care giver. Most of the respondents were not aware of anemia, bleeding, immunesuppression and loss of appetite induced by chemotherapy. This is probably because these are late effects and some health care givers confuse them as 
symptoms of disease itself. Similar trend of results were also reported by Lisa Ysela Ramirez et al in their study on Potential chemotherapy side effects, in their study they reported immediately occurring side effects as acute effect and others as late effects of chemotherapy 17

In a prospective cohort study on the incidence and severity of self-reported chemotherapy side effects in routine care, Alison Pearce et al revealed that chemotherapy side effects which are normally graded from grade 1 to 4 occurred in $86 \%$ of all participants of which $62 \%$ were moderate and severe side effects prompting them to seek medical care. This is slightly higher than that showed by our study of $88.4 \%$ signifying that the vast majority of patients on chemotherapy will suffer from significant side effects and this have been shown by many other studies ${ }^{\mathbf{1 8 , 2 0 , 2 1}}$. Having this knowledge, we expected participants who reported to have experienced side effect to take action. Among 191 respondents who experienced side effects, $66(34.6 \%)$ people went back to the doctor, 69 (36.9\%) tolerated while $56(29.3 \%)$ stopped the medication at least once. The difference of responses taken by participants was probably due to the fact that, the severity by which they experienced them was different, side effects were severe, moderately while to others they were mild. Usually management of chemotherapy side effect is a part of routine cancer management and patients are often told about management option available. Thus it was important to associate level of awareness and action taken by participants following the experience of side effect. There were a significant statistical association between the awareness of side effects and the action taken following the experience of side effects $(\mathrm{p}=0.021)$. Furthermore some participants $56(29.3 \%)$ of who stopped medication at least at once were not aware of side effects predicting poor outcome from their treatment. This is one of many reasons some patients abandon conventional treatment as shown by an observational study by Moshe Frenkel in his article titled "Refusing treatment" published on May 2013 in the Oncologist Journal and other studies 19, 20, 21, 22. Prior information on the chemotherapy side effects helps to make the patients aware and also promote adherence. It was also found that there was a significant statistical association between awareness to side effects and the duration in treatment $(\mathrm{p}=0.013)$, those who were on treatment for long duration of time were aware compared to those who has been on treatment for short time.

\section{Attitude towards chemotherapy use}

Attitude is a settled way of thinking or feeling about something, it is a single strong indicator driving an individual into willingly accepting something. Attitude can be changed by pre or post choice information. We found that most of participants, 91.2\% had positive attitude towards the use of chemotherapy. They said the use of chemotherapy was very helpful and their conditions have improved following the use of chemotherapy. Symptoms like swelling and excessive pain were reported to have decreased following the use of chemotherapy. These patients were so willing to continue receiving chemotherapy. The positive attitude showed by majority of participants was because of the benefit they gained after using them. However some respondents showed negative attitude and said that they did not find it beneficial to be on chemotherapy. This probably because, they received chemotherapy while their disease has been metastasized or they had wrong regimen of chemotherapy for a particular type of cancer. This rate of attitude is high similarly to multisite survey study on willingness to accept chemotherapy and attitude towards cost of cancer treatments done in Netherlands in which almost all patients accepted chemotherapy. Many studies show the attitude of patients towards chemotherapy to be positive ${ }^{\mathbf{2 3}, \mathbf{2 4 , 2 5 , 2 6}}$. There was a significant statistical association between awareness of side effects and attitude towards the chemotherapy use $(p=0.01)$. Those who were aware of the side effects had positive attitude while others the positive attitude were attributed by the benefit gained or expected following the use of chemotherapy.

\section{CONCLUSION}

The study found there was poor level of awareness of the chemotherapy side effects and higher level of positive attitude towards chemotherapy use among patients attending and receiving chemotherapy in oncology unit at Bugando Medical Centre. Only small portion of the respondents was aware of the chemotherapy side effects.

It was also found that those who were informed were aware only of few side effects. Moreover, the study revealed that unawareness of side effects affected adherence as most of the respondents who stopped medication were not aware of the side effects. It was also found that the pharmacists did not play their role to counsel and inform patients on the chemotherapy side effects.

\section{RECOMMENDATIONS}

The patients need to get informed thoroughly on the side effects of chemotherapy before they start treatment. Recorded videos giving relevant information are to be played in the rest room, but also the prepared brochures should be provided. We also recommend that other study to be done to assess the factors which might be impeding the transfer of information on side effects of chemotherapy to the patients.

\section{CONFLICT OF INTEREST}

Authors declare no conflict interests.

\section{Author's contributions}

Deogratias M Katabalo and Raphael Matinde conceived an idea and designed the study; they then along with Nestory Masalu collected data; Stanley Mwita and Karol Marwa analyzed the data; Deogratias Katabalo drafted the first manuscript; Stanley Mwita, Karol Marwa and Raphael Matinde critically reviewed the manuscript. 


\section{REFERENCES}

1. Organization WHO. Cancer Fact Sheet. 2014. Assesed at www.who.int/media centre/factsheets

2. Dictionary of Medical Terms. Fourth edition ed. London: A \& C Black Publishers Ltd; 2004.

3. Jemal A, Bray F, Center MM, Ferlay, Ferlay J, Ward E, Forman D, Global cancer statistics. A cancer journal for clinicians february 2011; 61(2):69-90.

4. Colin D Mathers, Dejan Loncar.Projection of Global Mortality and burden of disease from 2002 to 2030.PLoS Med Nov 2006,3(11):e442.

5. Foudation for cancer care in Tanzania. 2002 . Assesed at https://tanzaniacancercare.org

6. Kurt Ullman. Navigating Cancer treatment. Journal of the national cancer institute 106 (2) Feb 2014 https://doi.org/10.1093/jnci/dju031.

7. Julie Thompson, Kathryn Siliman, Dawn E Clifford. Impact of an early education multimedia intervention in managing nutritionrelated chemotherapy side effects. springerPlus. 2013; 2:179.

8. Benor DE, Delbar V, Krulik T. Measuring impact of nursing intervention on cancer patients' ability to control symptoms. Cancer nursing. 1998; 21(5):320-34.

9. Weeks JC, Catalano PJ, Cronin A, Finkelman MD, Mack JW, Keating NL, et al. Patients' expectations about effects of chemotherapy for advanced cancer. New England Journal of Medicine. 2012; 367(17):1616-25.

10. Carey MP, Burish TG. Etiology and treatment of the psychological side effects associated with cancer chemotherapy: a critical review and discussion. Psychological bulletin. 1988; 104(3):307.

11. Bugando medical Records, Oncology Unit 2017.

12. Maroun J.A, Blais N, Burkes R, Dranitsaris G, Shah A, Vincent M.D. Prevention and management of chemotherapyinduced diarrhea in patients with colorectal cancer: a consensus statement by the Canadian Working Group on ChemotherapyInduced Diarrhea. current oncolgy.14.

13. O'Brien BJ RJ, Rocchi A, Latreille J, Fine S, Vandenberg T and Laberge F. Impact of chemotherapy-induced nausea and vomiting on patients' functional status and costs: survey of five Canadian centers. cannada medical association. 1993; I49:296302 .
14. Lindley C.M, Hirsch J.D. Nausea and vomiting and cancer patients' quality of life: a discussion of professor Selby's paper. $\mathrm{Br}$ J Cancer 1992; 66:26-9.

15. Batchelor D. Hair and cancer chemotherapy: consequences and nursing care - a literature study. European Journal of Cancer Care. 2001; 10(3):147-63.

16. Margaret A Lyons, Mitchel M Shelton. Psychosocial impact of cancer in low- income rural/urban women: phase II. Online Journal for Rural Nursing Health Care. 2004; 4(2):27.

17. Freedman TG. Social and cultural dimensions of hair loss in women treated for breast cancer. cancer nursing. 1994; 17(4):33441.

18. Pearce A, Haas M, Viney R, Pearson SA, Haywood P, et al. Incidence and severity of self-reported chemotherapy side effects in routine care: A prospective cohort study. PLOS ONE (2017) 12(10)

19. Moshe Frenkel. Refusing treatment. J oncologist 2013; 18 (5):634-636

20. Dijk E.F.M.M, CoAykunturk M, Zuur A.T, Van der palen J, Van der Graaf W.T.A, Timmer-Bonte J.N.H, Wymenga A.N.M. Willingness to accept chemotherapy and attitudes towards cost of cancer treatment; A multisite survey study in Netherland. The Netherlands journal of medicine 2016.

21. Williams J WC, Cunningham-Warburton P. A narrative study of chemotherapy-induced alopecia. Oncology Nursing forum. 1999; 26(9):1463-8.

22. Maunsell E BC, Dubois L, Lauzier S, Fraser A. Work problems after breast cancer: an exploratory qualitative study. psycho-oncology. 1999; 8(6):467-73.

23. S R. experience of patients with chemotherapy-induced alopecia. Patient education and counseling. 2004; 52(3):333-9.

24. Meyers CA. How chemotherapy damages the central nervous system. Journal of biology( BMC). 2008;7:11.

25. William H. Redd GHM, Katherine N. DuHamel. Behavioral Intervention for Cancer Treatment Side Effects. Journal of the National Cancer Institute. 2001; 93:810-23.

26. Lindley CM HJ, O'Neill CV, Transau MC, Gilbert CS and Osterhaus JT. Quality of life consequences of chemotherapyinduced emesis. Qual Life Res. 1992; I:331-40. 